\title{
Environmental Impact Evaluation Method of Grinding Process Using Clustering and ANFIS
}

\author{
Yu-gang Wang ${ }^{1}$ and Shi-chao Xiu ${ }^{2}$ \\ ${ }^{1}$ School of Mechanical Engineering and Automation, Liaoning University of \\ Technology, 121001, China \\ ${ }^{2}$ School of Mechanical Engineering and Automation, \\ Northeastern University, 110819, China \\ ${ }^{1}$ E-mail: mech_teawang@163.com \\ ${ }^{2}$ E-mail: shchxiu@mail.neu.edu.cn
}

\begin{abstract}
Grinding process can cause resources depletion and environmental pollution seriously. However, the environmental impact evaluation of grinding process is rarely concerned and mentioned. In order to select the friendliest grinding process scheme on environment, this paper proposes a novel evaluation method using the improved FCM clustering and ANFIS. Compared with the traditional evaluation method, the proposed method features are as follows: the form of the ex-ante evaluation, the less subjective influence and the high intelligent degree. The improved FCM clustering algorithm, incorporating subtractive clustering and FCM clustering, is used to determine the clustering number automatically and generate the training sample set and testing sample set. Parameters of the evaluation model are modified with learning training samples, and the effectiveness is verified with testing samples. The testing results indicate that the environmental impact evaluation value can be calculated for each process scheme accurately, and the optimal selection of process schemes can be implemented effectively.
\end{abstract}

Keywords: grinding process; environment impact evaluation; subtractive clustering; FCM clustering; ANFIS

\section{Introduction}

The growing environmental concerns, the stricter legislative regulations, as well as the rising price of resource and energy, are significantly influencing the production way of manufacturing companies. Therefore, the whole manufacturing process need be redesigned and regulated in an environmentally friendly manner. It requires the various evaluation methods and tools to evaluate the environmental impact of manufacturing. However, it is difficult to evaluate the environmental impact of manufacturing process appropriately. Nowadays, the research on environmental impact evaluation has attracted more and more interest of the researchers.

Grinding is the most common form of abrasive machining. It has been applied extensively for the machining of workpiece with high size accuracy and superior surface finish [1-2]. However, the massive consumption of abrasives and grinding liquid, the emissions of toxic wastes and material, as well as the diffusion of micro powder and noise can pollute environment seriously in grinding process [3]. Moreover, wastes disposal, recycling, security measures must also be considered. Therefore, rational evaluation and optimal selection of the grinding process are contribute to environmental pollution reduction.

Due to the inaccuracy of evaluative index value and the complex correlation among evaluation indexes, the mapping relationship cannot be precisely expressed in linear mathematical model between the evaluation index and the evaluation result. This paper 
attempts to develop a novel method to evaluate the environmental impact based on the clustering algorithm and the artificial intelligent technology in the grinding process. The improved FCM clustering can determine the clustering number automatically and produce the sample set. ANFIS modifies parameters and learns rules based on training sample set. Furthermore, the effectiveness of the evaluation model is verified with testing sample set.

\section{Review of Literature}

At Present, the widely used practice for evaluating the potential environmental impacts primarily depends on two approaches: life cycle assessments (LCA) and analytic hierarchy process (AHP).

Life cycle assessment (LCA) is a technique for assessing the potential environmental impacts associated with a product, process, or service throughout its lifetime. LCAs have been used as a tool to identify "hot spots" in the production chain that may introduce opportunities for simultaneously lowering environmental impacts and improving efficiency and profitability [4]. Portha and others illustrated the potential of coupling LCA and exergy analysis in the petroleum industry. A naphtha catalytic reforming process had been selected and evaluated in terms of climate change, one of the classical environmental impacts used in LCA [5]. Umeda and others attempted to compare and design some modules by considering various life cycle attributes of the product, such as compatibility of materials, similarity of lifetime, maintenance cycle, and ease of disassembly [6]. Filleti and others overcame a bottleneck by a dynamic LCA of manufacturing processes, presented a method to perform a web based on dynamic life cycle inventory and impact assessment for manufacturing processes [7]. However, due to the deficiencies on collecting accurately LCA data at long time basis, gathering the data can be problematic, and the evaluation must be implemented at the end of lifetime of product or process. Therefore, LCA will not determine which product or process is the most cost effective or works the best in advance [8].

Analytic hierarchy process (AHP) method shows the process of determining the priority of a set of alternatives and the relative importance of attributes in a multi-criteria decision-making (MCDM) problem [9]. The primary advantage of AHP is the relative ease with which it handles multiple criteria and performs qualitative and quantitative data [10]. Heinemann and others presented a hierarchical approach and a case study for a synergetic combination of tools for simulating machine behavior and manufacturing line performance as well as for the calculation of total cost of ownership and environmental impacts of factory systems [11]. Ishizaka and Labib presented the group analytic hierarchy process ordering method: a new multi-criteria decision aid method for ordering alternatives in a group decision. Authors found four main advantages: significant reduction of time and effort in the decision process; easiness for the decision makers to arrive at a consensus; enhancement of the decision quality and documentation with justification of the decision made [12]. However, AHP is frequently criticized for its inability to adequately accommodate the inherent uncertainty and imprecision associated with mapping decision maker perceptions to extract number. It is difficult to prevent the evaluation result by using AHP from decision maker subjective preference [13].

\section{Evaluation Method}

In this study, the clustering extracts the features from the original input data, determines the clustering number automatically and generates the sample set. The evaluation model is built based on ANFIS, and evaluate environmental impact evaluation value for the process scheme with the sample set. Compared with the LCA method, the proposed method can make an evaluation in early stage of process development, and give a forecast result. Since there is no actual manufacturing of a prototype product in the planning stage, a forecast and evaluation method would be beneficial in terms of time and 
cost, based on information of the used material and manufacturing process. Compared with the AHP method, the proposed method avoids individual subjective judgment and favoritism by means of learning training samples, and guarantees the objectivity and consistency of the evaluation result. Therefore, the proposed method is better than the above two methods. Its features are as follows: the form of ex-ante evaluation, the less subjective influence and the high intelligent degree.

The block diagram of the proposed evaluation method based on clustering and ANFIS is shown in Figure 1.

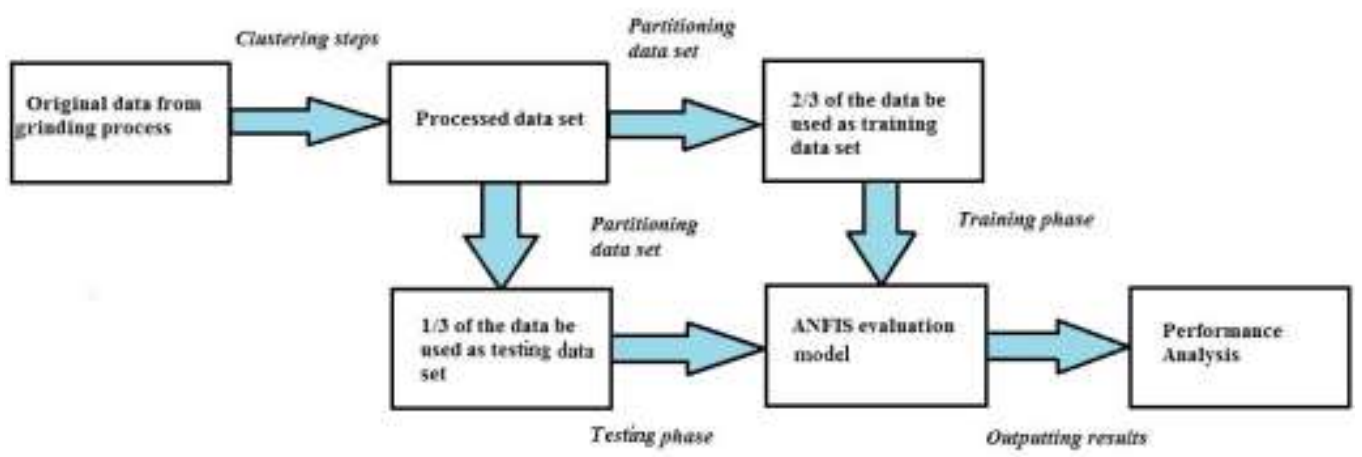

Figure 1. Procedures of the Evaluation Method

The method is divided into three steps which are described as fellows: The first step is to focus on the clustering analysis of the process data. The data set of optimal classification would be obtained by improved FCM clustering. The second step is to partition the classified data set: $2 / 3$ of the data are used for training sample set and 1/3 of the data are used for testing sample set. The third step is to design the evaluation model with the ANFIS. Evaluation model is trained by the training sample set to obtain the optimal system parameters, and then tested by the testing sample set to analyze the performance of evaluation results.

\subsection{Improved FCM clustering algorithm}

The FCM clustering is the most widely used the fuzzy clustering algorithm today. It has been widely researched and applied in the pattern recognition, the image processing, the fuzzy modeling, and other fields. However, the performance of FCM algorithm depends on the selection of the initial cluster center. The initial clustering center of FCM is generated randomly from the data set, and the clustering result is unstable. Besides, the clustering number of FCM also need to be determined in advance. Therefore, the improved clustering algorithm should be used to research the defects of FCM algorithm.

The subtractive clustering, a single fast algorithm, can estimate the clustering number and the cluster center of the data set automatically. In the subtractive clustering algorithm, each sample point is regarded as a potential cluster center. According to the data density around each sample point, the probability that the point becomes the cluster center is calculated. Using this method, the computation time is linearly proportional to the data size.

Therefore, the improving FCM algorithm with subtractive clustering not only has quicker convergence speed, but also effectively reduces the possibility of trapping in local optimal solution. Consider a collection of $n$ data points $\left(x_{1}, x_{2}, \ldots, x_{\mathrm{n}}\right)$ in a $\mathrm{P}$ dimensional space. The improved FCM algorithm is showed as follows:

1) Calculate a density cluster center $D_{\mathrm{i}}$ at data point $x_{\mathrm{i}}$ :

$$
D_{i}=\sum_{j=1}^{n} \exp \left[-\left\|x_{i}-x_{j}\right\|^{2} /\left(r_{a} / 2\right)^{2}\right]
$$


Where $r_{\mathrm{a}}$ - a positive constant representing a neighborhood radius; $\|*\|-$ the Euclidean distance between any measured data and the center.

2) Choose the data point with maximum density value as the first clustering center $D_{\mathrm{cl}}$. The potential value for each data point is revised as follows:

$$
D_{i}=D_{i}-D_{c 1} \exp \left(-\left\|x_{i}-x_{c 1}\right\|^{2} /\left(r_{b} / 2\right)^{2}\right)
$$

Where $r_{\mathrm{b}}$-a positive constant which defines a neighborhood radius that has significant reduction in density measure; $x_{\mathrm{cl}}$-the data point be selected as the first cluster center with the highest density value.

3) After the density measure of each data point is recalculated, next cluster center $x_{\mathrm{c} 2}$ is selected and all of the density measures for data points are revised again. This process will be terminated until the density value of all reminder data is less than the given threshold.

4) According to the results of subtractive clustering, the clustering number $C$ and clustering center $\mathrm{Z}(0)$ are determined. Set the weighted index, termination criterion $\varepsilon$ (value defined between 0 and 1), iteration count variable IT. The update of the membership degree matrix and the cluster centers are carried out through an iterative optimization of the objective function. It is based on the minimization of the following objective function:

$$
J\left(u_{i j}, z_{i}\right)=\sum_{i=1}^{C} \sum_{j=1}^{n} u_{i j}^{m}\left\|x_{j}-z_{i}\right\|^{2} \quad(m>1)
$$

Where $m$-any real number greater than $1, u_{\mathrm{ij}}$-membership degree of $x_{\mathrm{j}}$ in the $i^{\text {th }}$ clustering, $z_{\mathrm{i}}$ is the $i^{\text {th }}$ cluster center.

5) Calculating the membership degree matrix $U(I T)$.

$$
u_{i j}^{(I T)}=1 /\left[\sum_{k=1}^{C}\left(d_{i j} / d_{k j}\right)^{2 /(\lambda-1)}\right]
$$

Where $u_{\mathrm{ij}}$-membership degree of matrix elements; $d_{\mathrm{ij}}$-Euclidean distance of the $j^{\text {th }}$ sample to the $i^{\text {th }}$ cluster center. Membership degree $u_{\mathrm{ij}}$ must satisfy following constraints:

$$
\sum_{i=1}^{c} u_{i j}=1, \quad u_{i j} \in(0,1)
$$

6) Update the clustering center matrix $\mathrm{Z}(I T+1)$.

$$
z_{i}^{(I T+1)}=\left(\sum_{j=1}^{n}\left(u_{i j}\right)^{\lambda} \cdot x_{j}\right) / \sum_{j=1}^{n}\left(u_{i j}\right)^{\lambda}
$$

7) If $\left\|u_{i j}^{(I T+1)}-u_{i j}^{(I T)}\right\|\left\langle\varepsilon\right.$ or $I T<I T_{\max }$, then the iterative calculation will be terminated, else $I T=I T+1$, return to step 5 .

Where $I T_{\max }$-maximum termination times.

\subsection{ANFIS Evaluation Model}

ANFIS combines fuzzy logic and neural network and inherently possesses the advantages of both. ANFIS holds the learning abilities of ANN to enhance the intelligent inference system's performance, based on the gained knowledge after learning. Using a given training data set, ANFIS creates or improves a fuzzy inference system automatically whose membership function parameters are adjusted by hybrid type of the neural network algorithms.

The Takagi-Sugeno type structure of the ANFIS model is designed. The model is divided into two parts, namely Part I network including $\mathrm{C}$ subsystems ( $\mathrm{C}$ denotes clustering number) with four layers, and Part II network including $\mathrm{C}$ subsystems with three layers. The output value of the Part I network corresponds to the fitness value of the fuzzy rule, and the output value of the Part II network is applied to the weighted sum of 
the fuzzy rule. At last, the overall output of ANFIS is computed by mixing output values of two parts. In this paper, the ANFIS structure with P-dimension input and one-dimension output is as shown in Figure 2.

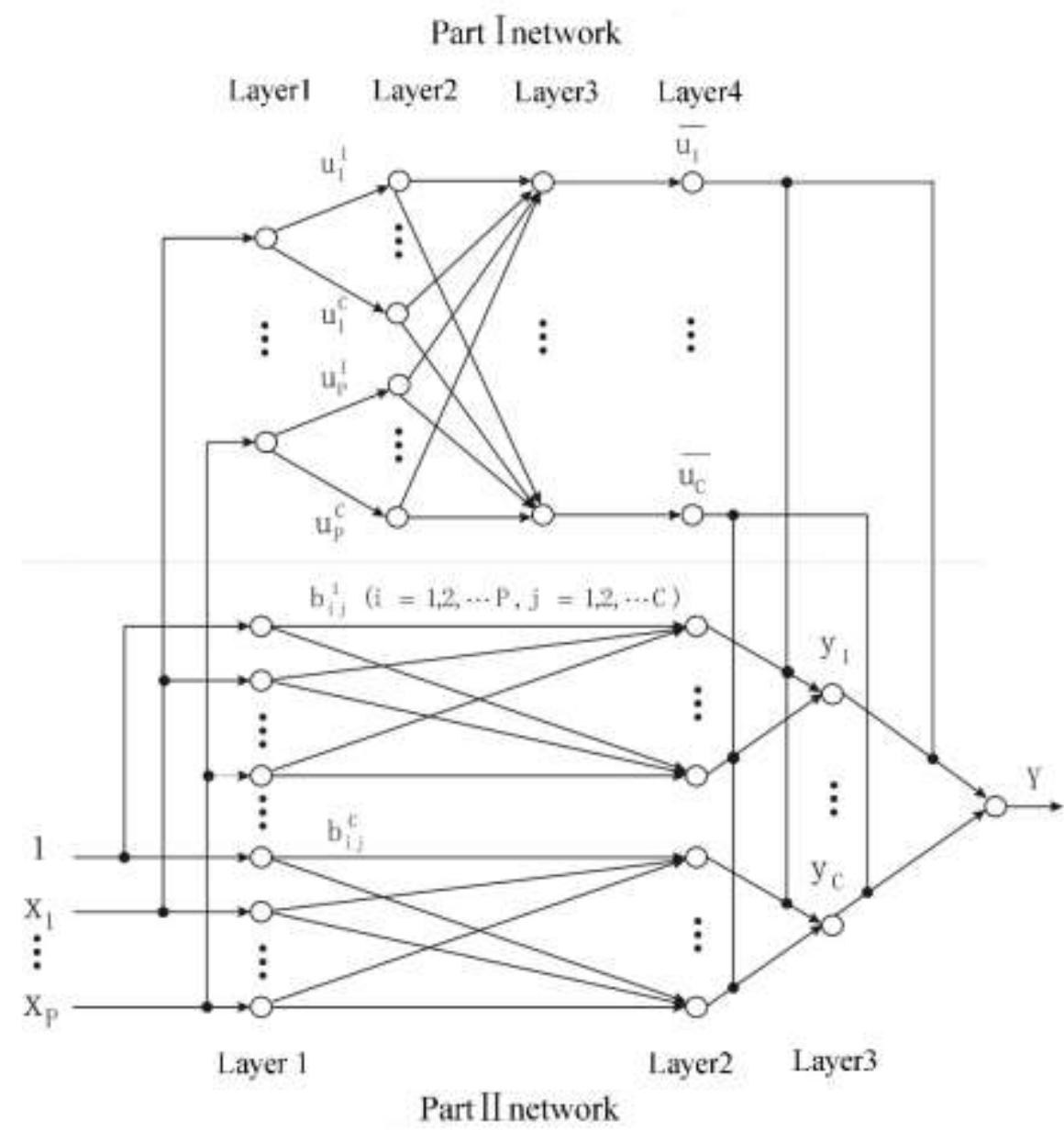

Figure 2. T-S Type ANFIS Structure

The Part I network subsystem is given as fellows. Layer 1 is called the 'input layer' where a linguistic label is associated with each input sample in terms of its membership grade. Layer 2 is known as a fuzzy layer. Each set of input values is converted by the corresponding fuzzy membership functions. Gaussian function is chose as the membership function. Layer 3 outputs the firing strength of the rule as the product of the membership grades. Layer 4 carries out normalization of firing strength of the rules. The output of the $i^{\text {th }}$ nodes equals the ratio of the $i^{\text {th }}$ rule's firing strength to the sum of all rules firing strength. The operation is also called normalization of firing weights:

$$
\overline{u_{i}}=\frac{u_{i}}{\sum_{i=1}^{C} u_{i}}
$$

Where $u_{i}$-firing weight of the $\mathrm{i}^{\text {th }}$ cluster center.

Brief narration about the Part II network subsystem is given as fellows. Layer 1 has the same function as the Part I network. Layer 2 is used to calculate the weight parameters $b_{i j}$ of each rule. Layer 3 represents the linear weighted sum of each rules. The output value $y_{i}$ $(i=1, \ldots, C)$ is calculated by using the output of the Part I network, i.e., the output of the Part I network is used as the third layer connection weights of the Part II network. 


$$
y_{i}=\sum_{i=1}^{C} \overline{u_{i}} y_{i j}
$$

At last, for a given input sample $\mathrm{X}=\left\{x_{1}, x_{2}, \ldots, x_{\mathrm{p}}\right\}$, the model overall output $Y$ can be shown by the following:

$$
Y=\sum_{i=1}^{C} \overline{u_{i}} y_{i}
$$

The learning algorithm of ANFIS is a hybrid algorithm that combines error back-propagation (BP) and recursive least square (RLS). The BP algorithm is used to adjust the parameter values of the Part I network. The RLS algorithm is used to optimize the connection weights of the Part II network.

In the running process, the model is initially established by the improved FCM clustering. In order to learn the experience from training samples, the hybrid algorithm is used to update the parameters of the model, until the output meets the error requirement or the number of iterations attains to the maximum.

The Root mean squared error (RMSE) between output value and expected value is used as a statistical indicator for evaluation criterion of the model. The lower the RMSE value is, the better the model is. Mathematically, RMSE is given as follows:

$$
R M S E=\sqrt{\sum_{j=1}^{n}\left[y(j)-y^{*}(j)\right]^{2} / n}
$$

Where $Y(j)$ - the model output value, $y *(j)$ — the model expected value.

\section{The Data}

\subsection{Data Collecting and Quantifying}

The data from the practical grinding process were used in this study. They were collected from the automobile manufacturing enterprise. It mainly included nine environmental impact evaluation indexes, as shown in Table 1:

Table 1. Grinding Process Evaluation Indexes

\begin{tabular}{ccc}
\hline $\begin{array}{c}\text { Index } \\
\text { No. }\end{array}$ & Index name & $\begin{array}{c}\text { Assigned value } \\
\text { method }\end{array}$ \\
\hline $\mathrm{X}_{1}$ & raw materials consumption $(\mathrm{Kg})$ & measured value \\
$\mathrm{X}_{2}$ & abrasive consumption (dimensional \\
change rate $)$ & measured value \\
$\mathrm{X}_{3}$ & solid waste $(\mathrm{Kg})$ & measured value \\
$\mathrm{X}_{4}$ & grinding fluid consumption $(\mathrm{L} / \mathrm{min})$ & measured value \\
$\mathrm{X}_{5}$ & grinding fluid toxicity & assigned value \\
$\mathrm{X}_{6}$ & dust & assigned value \\
$\mathrm{X}_{7}$ & pungent odor & assigned value \\
$\mathrm{X}_{8}$ & energy consumption $(\mathrm{kW} \cdot \mathrm{h})$ & measured value \\
$\mathrm{X}_{9}$ & Noise $(\mathrm{dB})$ & measured value
\end{tabular}

A common problem in evaluation data was how to describe the input value quantificationally. Because of the ambiguity of some index values, it was difficult to give 
the quantitative expression. In this study, the semi-quantitative method was used to quantify the evaluation indexes value. Evaluation indexes were divided into qualitative indexes and quantitative indexes. For quantitative indexes, measured values were directly used. For the qualitative indexes, the expert scored on a 10-point scale based on the specific regulation [14].

\subsection{Data Pre-processing}

The variable was transformed into an understandable or acceptable form before the training process. It was a kind of common practice. The process known as data pre-processing was carried out to reduce the dimensionality of input data and to optimize the generalization performance [15].

The input data was normalized by the following operation:

$$
x_{i}^{\prime}=\frac{x_{i}-x_{\min }}{x_{\max }-x_{\min }}
$$

Where $x_{\mathrm{i}}-\mathrm{a}$ required pre-processing data; $x_{\min }$-the minimum value of data set; $x_{\max }$ - the maximum value of data set.

The operation of pre-processing ensured that all input data were kept within the range $[0,1]$, so that the result values can be effectively analyzed.

\section{Model Test}

The pre-processing of 25 samples raw data had to be carried out before the model could be used. The final quantized values were shown in Table 2 .

Table 2. Quantized Values of Grinding Process Samples

\begin{tabular}{lccccccccc}
\hline No. & $\mathrm{x}_{1}$ & $\mathrm{x}_{2}$ & $\mathrm{x}_{3}$ & $\mathrm{x}_{4}$ & $\mathrm{x}_{5}$ & $\mathrm{x}_{6}$ & $\mathrm{x}_{7}$ & $\mathrm{x}_{8}$ & $\mathrm{x}_{9}$ \\
\hline 1 & 0.6 & 0.6 & 0.4 & 0.7 & 0.6 & 0.5 & 0.3 & 0.4 & 0.4 \\
2 & 0.2 & 0.2 & 0.4 & 0.5 & 0.4 & 0.3 & 0.5 & 0.2 & 0.5 \\
3 & 0.4 & 0.5 & 0.4 & 0.6 & 0.4 & 0.3 & 0.5 & 0.4 & 0.2 \\
4 & 0.8 & 0.8 & 0.6 & 0.6 & 0.6 & 0.5 & 0.7 & 0.6 & 0.5 \\
5 & 0.2 & 0.3 & 0.6 & 0.3 & 0.2 & 0.5 & 0.3 & 0.2 & 0.4 \\
6 & 0.3 & 0.3 & 0.6 & 0.5 & 0.2 & 0.5 & 0.3 & 0.4 & 0.5 \\
7 & 0.5 & 0.5 & 0.6 & 0.6 & 0.2 & 0.3 & 0.3 & 0.6 & 0.5 \\
8 & 0.4 & 0.6 & 0.4 & 0.7 & 0.2 & 0.5 & 0.7 & 0.4 & 0.4 \\
9 & 0.5 & 0.1 & 0.4 & 0.5 & 0.2 & 0.5 & 0.5 & 0.4 & 0.4 \\
10 & 0.6 & 0.5 & 0.6 & 0.5 & 0.2 & 0.3 & 0.7 & 0.6 & 0.6 \\
11 & 0.7 & 0.6 & 0.8 & 0.8 & 0.6 & 0.5 & 0.5 & 0.6 & 0.6 \\
12 & 0.8 & 0.7 & 0.6 & 0.8 & 0.4 & 0.7 & 0.3 & 0.6 & 0.5 \\
13 & 0.8 & 0.7 & 0.6 & 0.8 & 0.4 & 0.7 & 0.3 & 0.6 & 0.5 \\
14 & 0.6 & 0.6 & 0.8 & 0.6 & 0.8 & 0.5 & 0.7 & 0.4 & 0.5 \\
15 & 0.4 & 0.5 & 0.6 & 0.5 & 0.4 & 0.5 & 0.5 & 0.6 & 0.6 \\
16 & 0.6 & 0.5 & 0.4 & 0.6 & 0.4 & 0.5 & 0.3 & 0.6 & 0.6 \\
17 & 0.2 & 0.1 & 0.4 & 0.4 & 0.2 & 0.3 & 0.5 & 0.6 & 0.2 \\
18 & 0.3 & 0.3 & 0.6 & 0.3 & 0.4 & 0.5 & 0.3 & 0.2 & 0.5 \\
19 & 0.5 & 0.6 & 0.8 & 0.7 & 0.8 & 0.7 & 0.7 & 0.4 & 0.7 \\
20 & 0.4 & 0.5 & 0.4 & 0.5 & 0.4 & 0.3 & 0.3 & 0.4 & 0.5 \\
21 & 0.6 & 0.5 & 0.5 & 0.6 & 0.2 & 0.3 & 0.3 & 0.6 & 0.4 \\
22 & 0.7 & 0.7 & 0.6 & 0.6 & 0.8 & 0.5 & 0.5 & 0.8 & 0.7 \\
23 & 0.8 & 0.8 & 0.6 & 0.6 & 0.4 & 0.7 & 0.5 & 0.6 & 0.4 \\
24 & 0.4 & 0.2 & 0.4 & 0.4 & 0.4 & 0.3 & 0.5 & 0.5 & 0.2 \\
25 & 0.5 & 0.5 & 0.8 & 0.6 & 0.4 & 0.5 & 0.3 & 0.4 & 0.4 \\
\hline
\end{tabular}


MATLAB software was used for clustering analysis. By using the subtractive clustering with $r_{\mathrm{a}}=r_{\mathrm{b}}=0.6$, the clustering number $C=3$ was determined automatically. Under this condition, the FCM clustering and the improved FCM clustering were run respectively. The objective function curves were shown in Figure 3.

For the FCM clustering (red dashed lines), the training process was terminated after 17 iterations. However, for the improved FCM clustering (blue lines), the training process was decreased to 13 iterations. By comparing the results of two clustering methods, it was showed that the improved FCM clustering had faster convergence speed and better performance.

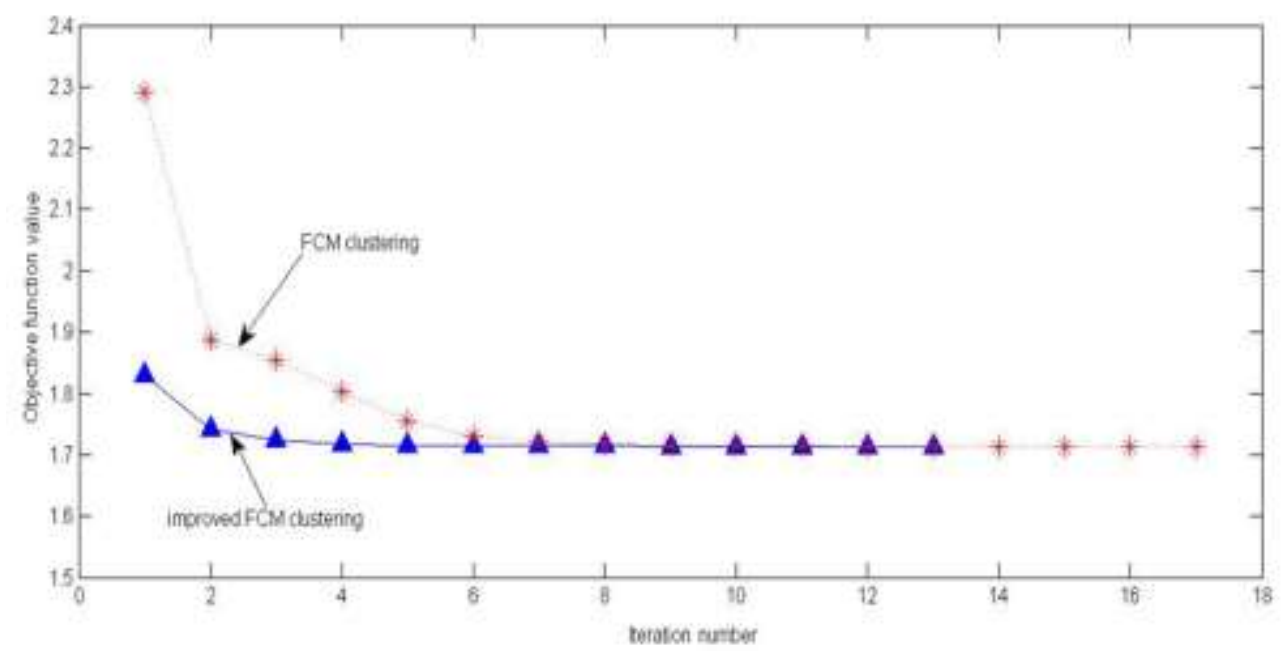

Figure 3. Objective Function Curves

The result of the improved FCM clustering was shown in Figure 4. The big black circle, triangle and star represented three clustering centers respectively.

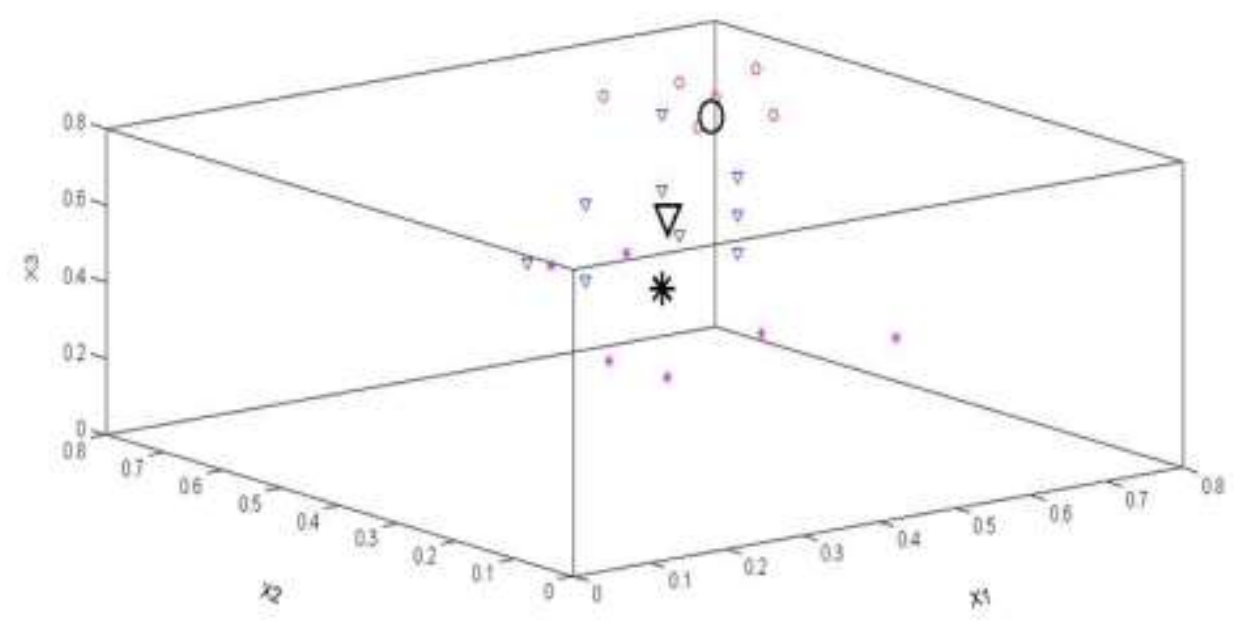

Figure 4. Result of Improved FCM Clustering

According to the weighted superposition method, the expected value (environmental impact evaluation value) of samples can be set as three level. When evaluation results were considered to be "excellent", the output expected value was set to 0.3. When evaluation results were considered to be "common", the output expected value was set to 
0.5. When evaluation results were considered to be "poor", the output expected value was set to 0.8. Therefore, the final results of samples clustering were shown in Table 3 .

Table 3. Result of Samples Clustering

\begin{tabular}{crcc}
\hline Clustering group No. & Sample No. & Expected value \\
\hline 1 & 2、5、6、9、17、18、24 & 0.3 \\
2 & 1、3、7、8、10、15、16、20、21、25 & 0.5 \\
3 & 4、11、12、13、14、19、22、23 & 0.8 \\
\hline
\end{tabular}

Before the evaluation model was used, the pre-processed data samples should be divided into the training sample set and the testing sample set. The samples with the number from 1 to 20 were assigned as the training samples, and the samples with the number from 16 to 25 as testing samples in Table 3 . The conditions of the simulation were set as follow: the maximum iteration number was 200 , the learning rate was 0.01 , and the fuzzy weighted index was 2.0. After training, the RMSE curve was obtained, and then used to ensure that the model did not cause over fitting. In the Figure 5, it can be seen that the RMSE curve became smooth and the value was very close to zero after 180 iterations. The observations showed that the performance of the evaluation model was good.

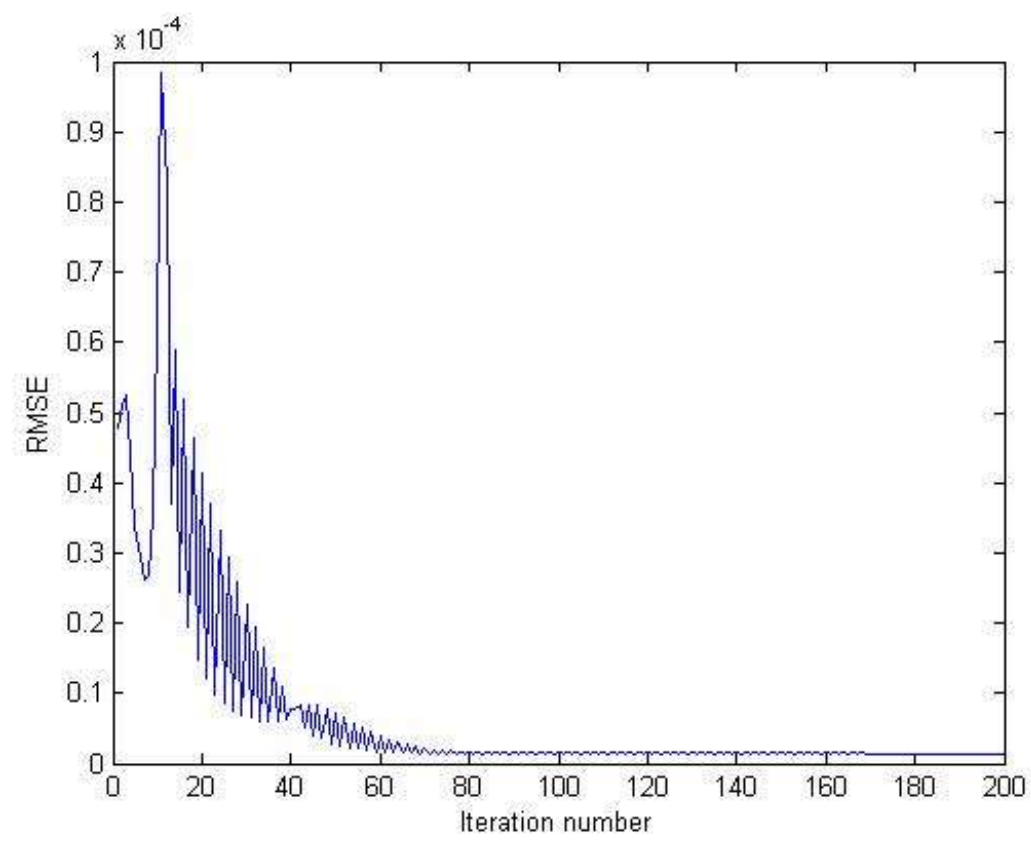

Figure 5. RMSE Curve of Training Samples

Finally, the testing samples were inputted into the model to calculate respectively. The distribution of output values and expected values was shown in Figure 6. 


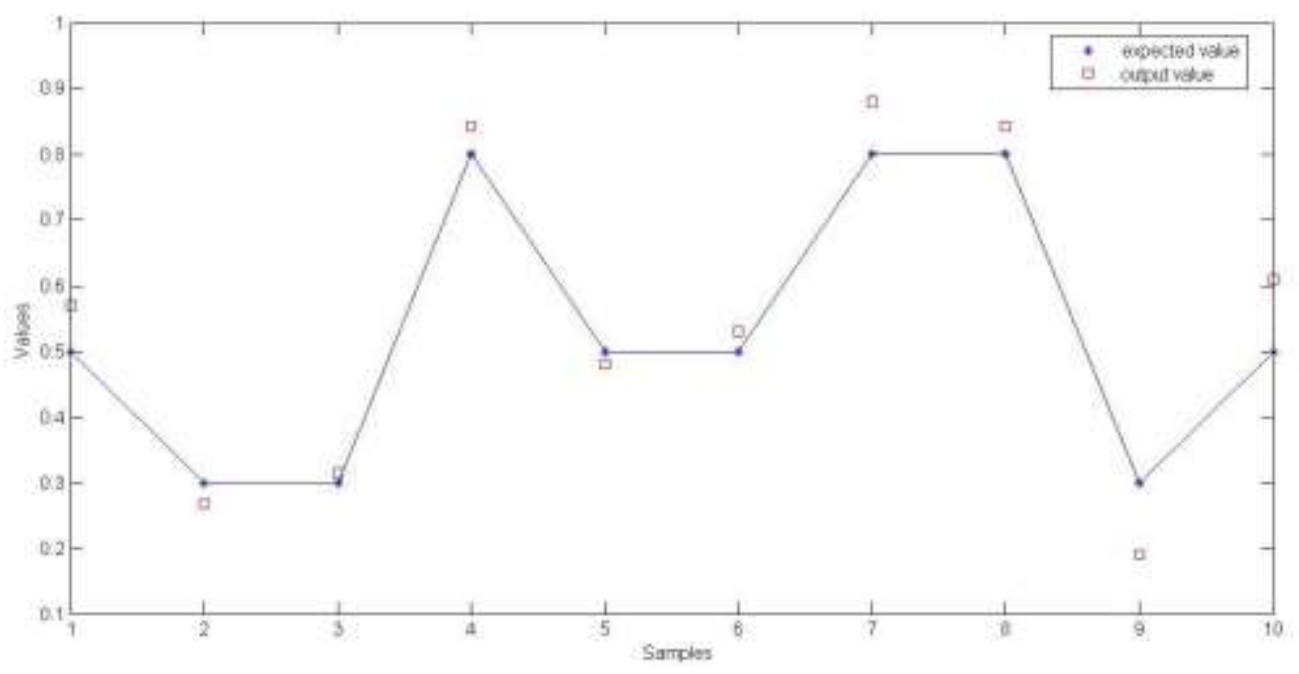

Figure 6. Output Values and Expected Values of Testing Samples

The Figure 6 showed comparing results of expected values with output values after testing. It can be seen that, by means of ten testing samples, the error of each input sample between the actual output and the expected output was very small in the evaluation model. In particular, the samples with the number from 21 to 25 were not applied to train evaluation model, but outputs were very close to the expectation. It indicated that the evaluation model can calculate environmental impact evaluation values accurately. Furthermore, testing results also indicated that the model had high prediction accuracy and intelligent analysis ability. The decision maker can identify the sorting and optimal selection of the process schemes based on environmental impact evaluation values.

\section{Conclusion}

The grinding process evaluation method has been presented. The evaluation model is established by incorporating clustering and ANFIS. The training sample set and the testing sample set are produced by means of the improved FCM clustering. Parameters of the evaluation model are modified by learning training samples, and the effectiveness is verified with testing samples.

Compared to previous investigations, the proposed method has the following contributions. First, the novel method with clustering and ANFIS is different from other evaluating methods, such as LCA and AHP. The method to evaluate environmental impact of process has never been found in previous literature. Second, the model shows its potential advantage in process scheme evaluation and optimal selection based on the testing result. It has following features: the form of the ex-ante evaluation, the less subjective influence and the high intelligent degree. Therefore, the method offers an effective way of selecting the optimal process to reduce environmental impact and save resource. Third, the proposed evaluation method can be widely used in the manufacturing. It is feasible to use the method to evaluate the green process, the green product, and the green material, etc.

\section{Acknowledgements}

Thanks are given to the Chinese national natural science fund committee. This project is supported by National Natural Science Foundation of China (Grant No. 51375083). The writer acknowledges the valuable comments and discussions with Mrs. Jin yanyan. 


\section{References}

[1] R. Alberdi, J. A. Sanchez, I. Pombo, N. Ortega, B. Izquierdo, S. Plaza, and D. Barrenetxea, "Strategies for optimal use of fluids in grinding", International Journal of Machine Tools \& Manufacture, vol. 51, no. 6, (2011), pp. 491-499.

[2] S. C. Xiu, M. H. Liu, C. X. Chao, and S. J. Li, "Study on micro-surface texture and tribology characters of ground surface in point grinding process", International Journal of Surface Science and Engineering, vol. 8, no. 2-3, (2014), pp. 225-238.

[3] C. H. Li, Q. Zhang, S. Wang, D. Jia, D. K. Zhang, Y. B. Zhang, and X. W. Zhang, "Useful fluid flow and flow rate in grinding: an experimental verification", International Journal of Advanced Manufacturing Technology, vol. 78, no. 5-8, (2015), pp.1 -10.

[4] D. Kim, G. Thoma, D. Nutter, F. Milani, R. Ulrich, and G. Norris, "Life cycle assessment of cheese and whey production in the USA", International Journal Life Cycle Assess, vol.18, no.5, (2013), pp. 1019 -1035 .

[5] J. F. Portha, S. Louret, M. N. Pons, and J. N. Jaubert, "Estimation of the environmental impact of a petrochemical process using coupled LCA and exergy analysis", Resources Conservation \& Recycling, vol. 54, no. 5, (2010), pp. 291-298.

[6] Y. Umeda, S. Fukushige, K. Tonoike, and S. Kondoh, "Product modularity for life cycle design," CIRP Ann., vol. 57, no. 1, (2008), pp. 13-16.

[7] R. A. P. Filleti, D. A. L. Silva, E. J. Silva, and A. R. Ometto, "Dynamic system for life cycle inventory and impact assessment of manufacturing processes", 21st CIRP Conference on Life Cycle Engineering, (2014), pp. 531-536.

[8] Scientific Applications International Corporation, "Life cycle assessment: principles and practice", http://www.epa.gov/ORD/NRMRL/lcaccess, (2006).

[9] C. C. Wei, C. F. Chen, and M. J. Wang, "An AHP-based approach to ERP system selection", International Journal of Production Economic, vol. 96, no. 1, (2005), pp. 47-62.

[10] C. Kahraman, U. Cebeci, D. Ruan, "Multi-attribute comparison of catering service companies using fuzzy AHP: the case of Turkey", International Journal of Production Economics, vol. 87, no. 2, (2004), pp.171-184.

[11] T. Heinemann, P. Schraml, S. Thiedea, C. Eiseleb, C. Herrmanna, and E. Abeleb, "Hierarchical evaluation of environmental impacts from manufacturing system and machine perspective", 21st CIRP Conference on Life Cycle Engineering, (2014), pp. 141-146.

[12] A. Ishizaka and A. Labib, "Selection of new production facilities with the group analytic hierarchy process ordering method", Expert Systems with Applications, vol. 38, no. 6, (2011), pp. 7317-7325.

[13] H. I. Lee, W. C. Chen, and C. J. Chang, "A fuzzy AHP and BSC approach for evaluating performance of IT department in the manufacturing industry in Taiwan”, Expert Systems with Applications, vol. 34, no. 1, (2008), pp. 96-107.

[14] Y. G. Wang, S. C. Xiu and K. Y. Wang, "Sample classification method for green process evaluation based on fuzzy clustering", Journal of Northeast University (Nature Science), vol. 37, no. 3, (2016), pp. 387-391.

[15] C. M. Bishop, "Neural networks for pattern recognition”, Oxford University Press, (2007), pp. 101-116.

\section{Authors}

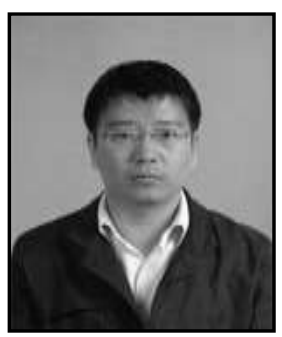

Yu-gang Wang, is an Associate Professor at Liaoning University of Technology in China. His research interests include green manufactures and high efficiency grinding technology. 
International Journal of Control and Automation Vol.10, No.8 (2017) 